\title{
Saúde mental em concursos de residência médica: implicações das Diretrizes Nacionais Curriculares de 2014
}

\author{
Mental health in medical residency admission tests: implications of the 2014 National Curriculum Guidelines \\ Julia Melara' (1) juliamelara@gmail.com \\ Gustavo Gomedi' (1) gustavogomedi@yahoo.com \\ Felipe Pinheiro de Figueiredo' (D) felipe.figueiredo@unicesumar.edu.br
}

\begin{abstract}
RESUMO
Introdução: Devido à importância da saúde mental (SM) na prática médica, as Diretrizes Curriculares Nacionais do Curso de Graduação em Medicina (DCN) de 2014 estabeleceram a valorização desse tema. Embora as provas de residência médica (RM) devam ser influenciadas por essa diretriz, desconhecia-se se elas estavam de acordo com as mudanças.

Objetivo: Este trabalho teve como objetivo avaliar se as diretrizes determinadas pelo Ministério da Saúde têm sido seguidas, buscando a devida valorização do tema SM.

Método: Realizou-se uma pesquisa documental, sobre como a SM vem sendo abordada nos concursos de RM, na modalidade acesso direto, antes e depois da publicação das DCN. Para isso, selecionaram-se, por conveniência, nove instituições do Sul e Sudeste do Brasil, delimitando-se um período de dez anos para análise. Dois investigadores realizaram a seleção e análise das provas e das questões. Decisões em casos de discordância foram feitas a partir da avaliação de um terceiro investigador (juiz). Após esse processo, categorizaram-se as questões em subtemas, e tabularamse os dados em uma planilha, analisada descritivamente com distribuições percentuais para variáveis qualitativas e medidas de tendência central.
\end{abstract}

Resultado: Os conteúdos de SM corresponderam a 3,8\% das provas. Não foi constatado crescimento na porcentagem de questões consequente à publicação das DCN. Verificou-se o predomínio das questões com essa temática nas áreas de clínica médica e de saúde coletiva $(n=129 / n=109)$, contrapondo-se à cirurgia geral $(n=0)$. Houve uma superioridade do subtema "psicofarmacologia" $(n=102)$.

Conclusão: Como visto, no que tange à SM, as DCN não alcançaram ainda os concursos de RM. O enfoque da SM dado às questões das instituições analisadas permanece com uma visão biologicista.

Palavras-chave: Internato e Residência; Saúde Mental; Psiquiatria Comunitária.

\begin{abstract}
Introduction: Due to the importance of Mental Health $(\mathrm{MH})$ in medical practice, the National Curriculum Guidelines for the Undergraduate Course in Medicine (DCN) published in 2014 established inclusion of this subject matter. Although medical residency tests must have been affected by this resolution, it remained unclear whether those tests were in line with the new guidelines.
\end{abstract}

Objective: this study aims to assess whether the guidelines determined by the Ministry of Health are being followed and, thus, giving due appreciation of the theme of $\mathrm{MH}$.

Method: Documentary research was conducted on how MH has been approached in the "Direct Access" Medical Residency (RM) admission tests, before and after the publication of the DCN. For this purpose, nine institutions in the south and southeast of Brazil were selected for convenience, delimiting a 10-year period for analysis. The selection, analysis of the evidence and questions were developed by two researchers. In the event of disagreement, decisions were made based on the evaluation of a third investigator (judge). After this process, the questions were categorized into subtopics and the data tabulated on a spreadsheet, analyzed descriptively with percentage distributions for qualitative variables and measures of central tendency.

Result: The MH content corresponded to $3.8 \%$ of the tests. There was no increase in the percentage of issues resulting from the publication of the DCNs. There was a predominance in the areas of Clinical Medicine and Public Health ( $n=129 / n=109)$, in contrast to "General Surgery" ( $n=0)$. Superiority of the subtopic "Psychopharmacology" was found ( $n=102)$.

Conclusion: As described, there are not yet any implications of the DCN found in the MR admission tests in relation to MH. The way MH is approached in the test paper questions of the analyzed institutions continues to reflect a biological perspective.

Keywords: Internship and Residency; Mental Health; Community Psychiatry.

${ }^{1}$ Universidade Cesumar, Maringá, Paraná, Brasil.

Editora-chefe: Rosiane Viana Zuza Diniz.

Editor associado: Pedro Tadao Hamamoto Filho.

Recebido em 30/01/21; Aceito em 25/05/21.

Avaliado pelo processo de double blind review. 


\section{INTRODUÇÃO}

Nos últimos 30 anos houve um aumento de faculdades de Medicina no Brasil, levando à crescente dificuldade de controle da qualidade dos egressos e dos cursos. Sabe-se que os modelos curriculares, o preparo de médicos para a docência e os contextos de práticas adequados ao ensino são fatores relevantes para a qualidade da formação médica'. Assim, o modelo curricular tradicional, ao focar disciplinas biológicas e independentes, pode ser desfavorável ao desenvolvimento de autonomia do aluno, integração de conhecimentos, análise, julgamento e avaliação, bem como ao raciocínio crítico, investigativo e criativo ${ }^{1,2}$.

A abertura de escolas médicas com a velocidade que aconteceu no Brasil propiciou alguns desafios. Por um lado, temos médicos sem um correspondente preparo para o ensino e inexperientes em pesquisa, apresentando-se com um preparo reflexivo aquém do necessário para a docência ${ }^{3}$. Por outro lado, a abertura de escolas médicas prescinde do preparo de contextos de práticas adequados ao ensino para a execução de cursos com uma boa carga horária de prática. Se esses desafios não forem adequadamente observados, debates e indagações acerca da qualidade da educação médica brasileira poderão vir à tona, e teremos uma comunidade insatisfeita e médicos despreparados para os desafios impostos pela profissão $0^{3,4}$.

Objetivando atuar sobre esse quadro, a Resolução do Conselho Nacional de Educação e da Câmara de Educação Superior (CNE/CES) no 3, de 20 de junho de 2014 instituiu as Diretrizes Curriculares Nacionais do Curso de Graduação em Medicina (DCN), a serem observadas na organização, no desenvolvimento e na avaliação dos cursos de Medicina ${ }^{5}$. Assim, as DCN passaram a estabelecer os princípios, as metodologias, as condições, os procedimentos e as finalidades dessa formação. A partir disso, a estrutura do curso de graduação em Medicina deveria articular: teoria e prática; instituições formadoras e prestadoras de serviços; distintas áreas de conhecimento; e aspectos objetivos, subjetivos e conjunturais. $\mathrm{O}$ processo de formação visa a médicos propositivos e resolutivos, com visão humanista, crítica, reflexiva e ética, capazes de relacionar o processo saúde-doença nos diferentes níveis de atenção. Entre as formas de se alcançar os objetivos, as DCN estabeleceram as seguintes áreas de práticas do estágio curricular obrigatório: atenção básica, urgência e emergência, clínica médica, clínica cirúrgica, ginecologia e obstetrícia, pediatria, saúde coletiva e saúde mental (SM).

Assim, a partir das DCN de 2014, a SM passou a ser uma área crucial para a formação médica, assumindo papel comparável a áreas tradicionais do currículo médico. Isso se baseia na crescente prevalência da SM e nos impactos em morbidade para a população acometida por transtornos mentais. Essa população, por sua vez, enfrenta grandes problemas assistenciais motivados, em parte, por atitudes estigmatizadoras advindas de profissionais de saúde. Estágios práticos curriculares de curta duração ou com pouco aprofundamento sustentam essas atitudes ${ }^{6-8}$. Por ser a graduação um período crucial para promoção de mudanças atitudinais, a experiência particular do aluno de Medicina no tocante à SM depende de aspectos do currículo das faculdades, como o foco dado à psiquiatria e a áreas afins, bem como o apoio do corpo docente da área ${ }^{9}$. Dessa forma, a evidência dada à SM na graduação em Medicina busca, em última análise, uma melhoria assistencial ao público acometido com esse tipo de agravo.

Como complemento à graduação médica, a modalidade padrão-ouro do lato sensu de pós-graduação médica é a residência médica (RM), instituída pelo Decreto no 80.281 , de 5 de setembro de $1977^{10}$. Diante da grande concorrência e de constantes imposições por atualização e refinamento exigidas pelo atual mercado de trabalho, a RM constitui parte do processo recomendado de formação médica, sendo, para os estudantes, o caminho óbvio após o internato. Essa preocupação se exacerba ainda mais porque existe uma maior proporção de graduandos em Medicina do que vagas de ingresso em Programas de Residência Médica (PRM) ${ }^{4}$.

De forma geral, os PRM contam com uma seleção pública dos candidatos por meio de exame escrito, obrigatório, objetivo, com igual número de questões nas especialidades de clínica médica, cirurgia geral, pediatria, ginecologia e obstetrícia, medicina preventiva e social (saúde coletiva e medicina geral de família e comunidade). A segunda fase, opcional, a critério da instituição, é constituída de prova prática equivalente a até $50 \%$ da nota total ${ }^{11}$.

Como a prova teórica tem grande peso para o ingresso na RM e a concorrência do processo é elevada, surgem os cursos preparatórios, os quais utilizam um método simples, objetivo e resumido, que, ao revisarem os conteúdos mais prevalentes nas provas de RM, acabam distanciando o conteúdo teórico do prático aprendido nos estágios supervisionados. Isso faz com que os alunos estudem mais o primeiro em detrimento do segundo, conforme há um aumento na necessidade da carga horária para estudo. Tal fato prejudica a formação dos alunos que se afastam das atividades clínicas, comprometendo a assimilação de conceitos e atitudes importantes, como a relação médico-paciente e habilidades na entrevista médica. Para áreas pouco cobradas nas provas de RM, a importância dada pelo aluno do internato torna-se ainda menor ${ }^{4}$.

Portanto, torna-se importante analisar pormenorizadamente as provas que intermedeiam o ingresso do aluno nos PRM, visto que elas deveriam se basear nos 
conteúdos curriculares desenvolvidos durante o processo de graduação, que, por sua vez, deveriam se basear nas DCN. Dada a importância do enfoque em SM para a graduação médica como forma de mudança de paradigmas e políticas assistenciais, analisar essa área, especificamente, pode ser relevante como forma de avaliar se as provas de ingresso em PRM estão contemplando as mudanças contidas nas DCN.

\section{MÉTODO}

Foi realizada uma pesquisa documental, em que se analisaram provas de concursos de RM na modalidade acesso direto. Delimitou-se um período de dez anos (de 2009 a 2019) para análise, e padronizaram-se essas provas de acordo com o ano em que os residentes foram admitidos nos respectivos processos seletivos. Desse modo, a Associação Médica do Rio Grande do Sul (AMRIGS) e a Universidade Federal do Paraná (UFPR) tiveram as provas de 2008 corrigidas. Por conveniência, selecionaram-se concursos de instituições do Sul e Sudeste do Brasil, com reconhecida importância na formação médica, a saber: Universidade de São Paulo - câmpus São Paulo (USPSP), Universidade de São Paulo - câmpus Ribeirão Preto (USP-RP), Universidade Estadual de Campinas (Unicamp), Universidade Federal do Rio de Janeiro (UFRJ), Universidade Federal de São Paulo (Unifesp), UFPR, Universidade Estadual de Maringá (UEM), AMRIGS e Sistema Único de Saúde de São Paulo (SUS-SP).

As provas foram adquiridas por meio dos próprios sites das universidades, sites de cursinhos preparatórios para as provas de RM, bem como nos principais bancos de provas do país. As provas não encontradas por esses meios foram buscadas por comunicações eletrônicas diretas às comissões de RM dessas instituições. Dois investigadores realizaram a seleção e análise das provas e das questões. Decisões em casos de discordância foram feitas a partir da avaliação de um terceiro investigador (juiz). Para o estudo, selecionaram-se as questões que contemplaram os conteúdos referentes à SM, considerando a psiquiatria como uma área de estudo dentro da SM. Incluíram-se questões anuladas pelos concursos.

Após seleção das questões, elas foram categorizadas dentro das cinco grandes áreas da Medicina: clínica médica, cirurgia geral, pediatria, obstetrícia e ginecologia e saúde coletiva (medicina preventiva e social). Posteriormente, foram classificadas nos seguintes subtemas: 1 . emergências em psiquiatria, 2. transtornos somatoformes, dissociativos e factícios, 3. transtornos alimentares, 4. Psiquiatria infantojuvenil, 5. esquizofrenia e outros transtornos psicóticos, 6. transtornos de personalidade, 7. transtornos de ansiedade, 8. transtornos mentais orgânicos (secundários a uma condição médica geral), 9. psicofarmacologia, 10. transtornos do humor, 11. transtornos mentais decorrentes de substâncias psicoativas, 12. Psicologia médica, 13. transtornos relacionados ao estresse, 14. Psiquiatria comunitária, saúde coletiva e atenção primária, 15. transtornos do neurodesenvolvimento, 16. transtornos mentais comórbidos a uma condição médica, 17. transtornos do controle de impulsos e 18. outros transtornos não descritos.

Algumas questões foram categorizadas em mais de um subtema. Convencionou-se a categorização das questões que abordaram o tema "maus-tratos na infância" como pertencentes à psiquiatria infantojuvenil. Aquelas que abordaram temas relacionados à violência e a outros fatores estressores no adulto foram incluídas no grupo dos transtornos relacionados ao estresse.

Tabularam-se os dados em uma planilha, a partir da qual foi realizada a análise descritiva com distribuições percentuais para variáveis qualitativas e medidas de tendência central.

\section{RESULTADOS}

De 99 provas, dez (10,1\%) não foram analisadas, por não estarem disponíveis nos meios de busca delimitados para este trabalho. Os concursos da UEM foram os que tiveram maior quantidade de indisponibilidades (sete provas, de 2009 a 2015). As demais instituições que não tiveram provas analisadas foram: SUS-SP (concurso de 2009), Unicamp (concurso de 2009) e USP-SP (concurso de 2015).

Verificaram-se ao total 8.508 questões, sendo: UFRJ 1.080 questões; UFPR - 1.100; Unifesp - 1.153; Unicamp - 800; USP-SP - 755; USP-RP - 1.100; SUS-SP - mil; UEM - 400; AMRIGS1.100. Desse total, foram encontradas 329 questões com conteúdos relacionados à psiquiatria e SM, correspondendo a aproximadamente $4 \%$ (3,8\%) das provas.

Observaram-se 64 questões sobre o tema nas provas da USP-SP, sendo a instituição com maior número de questões em SM, seguida pela AMRIGS ( $n=51)$ e Unifesp $(n=39)$. A instituição que obteve menor número que questões de $S M$ nos concursos foi a UFPR $(n=26)$.

O Gráfico 1 expressa os percentuais das questões com conteúdo referentes à psiquiatria e SM segundo as diferentes instituições, de acordo com os anos analisados.

Agrupamos as instituições por proximidade de administração para analisar, ano a ano, o número de questões em SM. Para realizar essa análise, agruparam-se as universidades federais (UFRJ, UFPR, Unifesp), as universidades estaduais de São Paulo (USP-SP, USP-RP e Unicamp) e, em outro conjunto, as demais instituições (SUS-SP, AMRIGS e UEM).

Diante disso, constata-se tendência de crescimento nas porcentagens de questões, no período de 2015 a 2018, das instituições dos grupos das estaduais de São Paulo e outras, no entanto as instituições federais não seguem esse padrão. 
Gráfico 1. Porcentagem de questões em SM encontradas por ano nas provas de RM por instituição ao longo dos anos analisados.

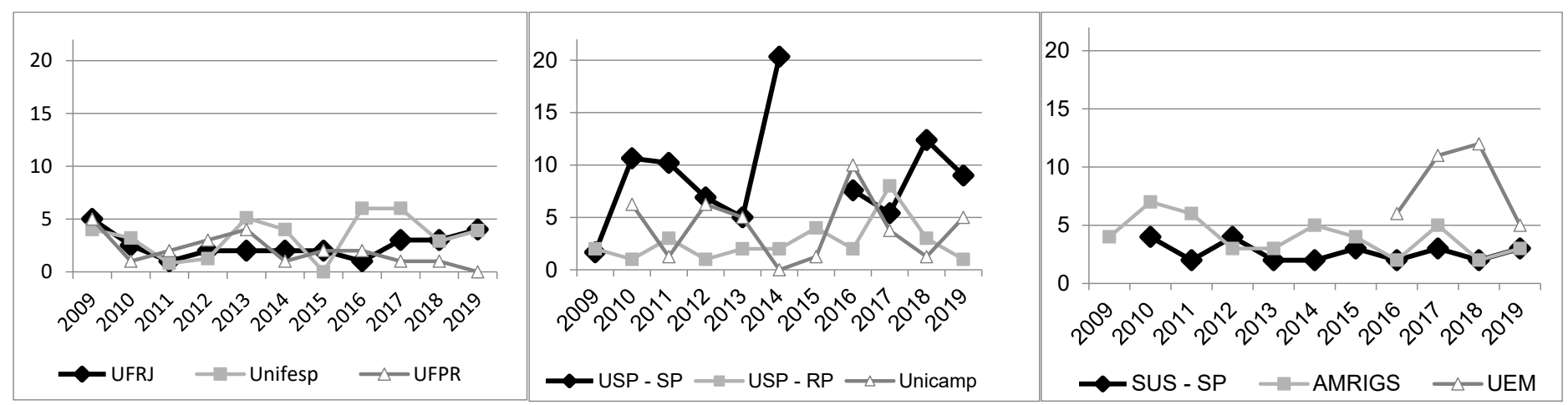

Fonte: Elaborado pelos autores com base na análise de provas de RM - acesso direto.

Gráfico 2. Porcentagem de questões, em relação ao total, encontradas em SM por ano, de acordo com o grupo de instituições citadas anteriormente.

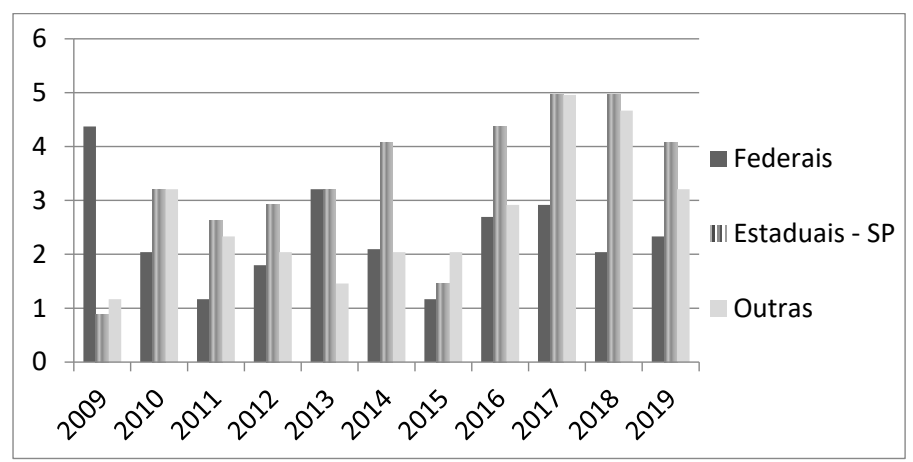

Fonte: Elaborado pelos autores com base na análise de provas de $\mathrm{RM}$ - acesso direto.
Não obstante, o ano de 2019 indica uma inclinação para uma nova estabilização.

Quando analisaram as questões de SM encontradas, verificou-se um predomínio de questões nas seguintes grandes áreas da Medicina: clínica médica e saúde coletiva. Em quatro das nove instituições, as questões de SM sobressaíram em coletiva: UFPR, UEM, USP-SP e AMRIGS. A subárea em que a SM é menos encontrada nas provas de residência é cirurgia geral, uma vez que nessa não foi encontrada nenhuma questão envolvendo esse tema em nenhuma prova, de nenhum ano ou instituição.

A posteriori, as questões de SM identificadas foram classificadas em subtemas, de acordo com seu conteúdo (Gráfico 3). Nota-se que o tema em SM que mais predominou foi "psicofarmacologia" ( $n=102)$. Em seguida, constam, nesta ordem:

Tabela 1. Quantidade e porcentagem de questões encontradas em SM por área da Medicina, entre as instituições analisadas.

\begin{tabular}{ccccccc}
\hline \multicolumn{7}{c}{ ÁREAS DA MEDICINA } \\
\hline INSTITUIÇÃO & CM [n (\%)] & GO [n (\%)] & PED [n (\%)] & CIR [n (\%)] & SC [n (\%)] & TOTAL [n (\%)] \\
\hline UFPR & $3(11,5)$ & $4(15,4)$ & $7(27)$ & 0 & $12(46,1)$ & $26(100)$ \\
UFRJ & $13(48,1)$ & $3(11,1)$ & $7(25,9)$ & 0 & $4(14,9)$ & $27(100)$ \\
Unifesp & $24(61,5)$ & $2(5,1)$ & $10(25,7)$ & 0 & $3(7,7)$ & $39(100)$ \\
Unicamp & $12(37,5)$ & $5(15,6)$ & $6(18,7)$ & 0 & $9(28,2)$ & $32(100)$ \\
USP-SP & $24(37,5)$ & $6(9,4)$ & $7(10,9)$ & 0 & $27(42,2)$ & $64(100)$ \\
USP-RP & $16(55,1)$ & $3(10,4)$ & $3(10,4)$ & 0 & $7(24,1)$ & $29(100)$ \\
AMRIGS & $9(17,7)$ & $3(5,9)$ & $8(15,6)$ & 0 & $31(60,8)$ & $51(100)$ \\
UEM & $12(35,2)$ & $6(17,6)$ & $3(8,9)$ & 0 & $13(38,3)$ & $34(100)$ \\
SUS-SP & $16(59,2)$ & $5(18,6)$ & $3(11,1)$ & 0 & $3(11,1)$ & $27(100)$ \\
\hline TOTAL [n (\%)] & $129(39,2)$ & $37(11,2)$ & $54(16,5)$ & 0 & $109(33,1)$ & \\
\hline
\end{tabular}

$\mathrm{CM}$ = clínica médica; $\mathrm{GO}$ = ginecologia e obstetrícia; $\mathrm{PED}=$ pediatria; $\mathrm{CIR}$ = cirurgia geral; $\mathrm{SC}$ = saúde coletiva.

Fonte: Elaborada pelos autores com base na análise de provas de RM - acesso direto. 
Gráfico 3. Comparação geral, independentemente de instituição ou ano, dos subtemas relacionados à SM encontrados nas questões avaliadas.

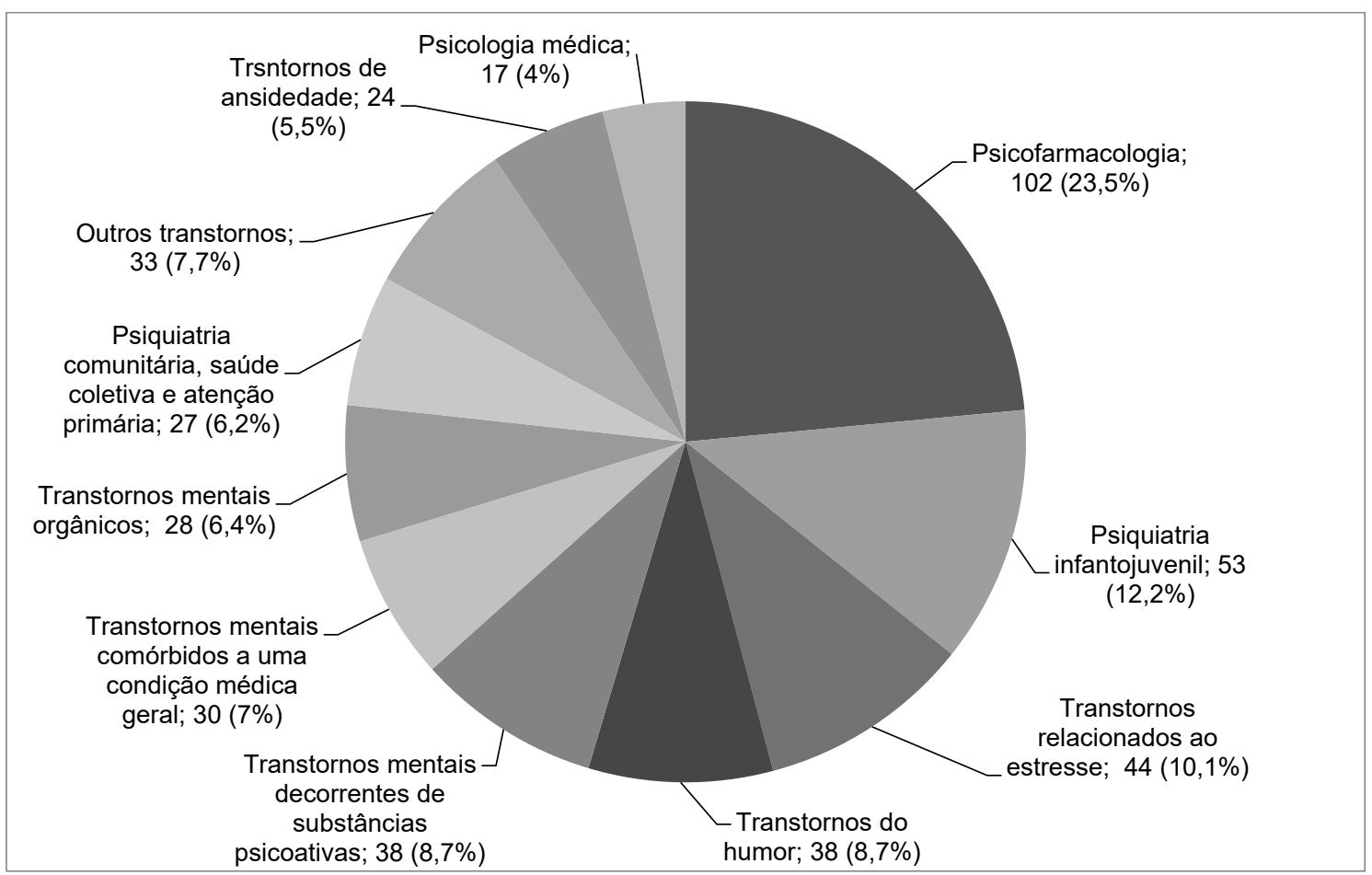

Fonte: Elaborado pelos autores com base na análise de provas de RM - acesso direto.

"psiquiatria infantojuvenil" ( $\mathrm{n}=53$ ), "transtornos relacionados ao estresse" ( $n=44)$, "transtornos do humor" ( $n=38)$ e "transtornos mentais decorrentes de substâncias psicoativas" $(n=38)$.

A predominância sobre o tema de psicofarmacologia aconteceu em todas as instituições, com exceção da USP-SP, na qual a maior carga ficou com o tema "transtornos relacionados ao estresse".

\section{DISCUSSÃO}

Com os resultados obtidos, verificou-se que nenhuma instituição analisada apresentou um aumento nas questões relacionadas à SM após a publicação das DCN em 2014. Entre as instituições e provas analisadas, o percentual de questões variou de $2 \%$ a 2,8\% antes de 2014 e de 1,5\% a 4,2\% após 2014. Assim, constatou-se que os concursos de RM, na modalidade acesso direto, ainda não acompanham as DCN, contemplando o aumento da carga horária prevista para o internato médico na área da SM.

As DCN têm como um de seus objetivos oferecer um direcionamento para a graduação em Medicina. Os PRM são complementos à formação médica. Tendo em vista a concorrência cada vez maior para o ingresso nos PRM, os concursos de RM são cada vez mais levados em consideração pelo graduando em Medicina como um guia para sua formação. Assim, indiretamente, as DCN deveriam influenciar o conteúdo dessas provas. Diante disso, a análise e o acompanhamento das provas de RM são importantes como forma de avaliar a implantação do exposto nas DCN. Diante da relevância, as mudanças sugeridas sobretudo na área de SM, devem ser continuamente acompanhadas. Pelo exposto, a abordagem de temas relacionados à SM nos PRM ainda se encontra muito aquém do necessário para ampliar o interesse dos alunos pelo tema.

Um dos fatores que contribuem para os dados visualizados nesta pesquisa, como a diminuta quantidade de questões em SM, restritas principalmente a apenas duas áreas médicas, é o fato de ainda não haver um acordo em relação a um formato eficiente de treinamento de residentes em psiquiatria com o enfoque no cuidado integral da SM. No Brasil, esse treinamento está limitado a hospitais terciários e de ensino que contêm unidades de internação e ambulatório ${ }^{12}$, o que amplia a segmentação entre a SM e as outras áreas da Medicina. Visto isso, a Associação Psiquiátrica Mundial, em parceria com a Federação Mundial de Educação Médica, definiu mundialmente um Currículo Principal em Psiquiatria, especificando atitudes, conhecimentos e habilidades que os graduandos devem portar para futuramente tratar doenças mentais. Contudo, de acordo com Thomas et al. ${ }^{13}$, a ausência de modelos curriculares atualizados e pesquisas práticas atuais na literatura recente sustenta que apenas a determinação teórica do currículo ideal não é suficiente para que os alunos vivenciem, no cotidiano, as mudanças estabelecidas. 
Por esses motivos, buscando expandir as experiências de aprendizagem dos residentes no SUS, por meio da multiplicidade de contextos de aprendizagem, a Secretaria de Saúde da cidade de São Paulo lançou em 2018 um novo modelo de RM integrada em psiquiatria, que ainda se encontra em processo de aperfeiçoamento ${ }^{12}$. O novo programa é realizado em três anos, contando com 60 horas de treinamento por semana divididos em porcentagens distintas a cada cenário, seguindo as recomendações da Comissão Nacional de Residência e do Ministério da Educação. Nessa estruturação inédita, os PRM adotam hospitais, ambulatórios e rede de atenção psicossocial (RAPS), com foco na atenção primária ${ }^{12}$.

Essa transformação fornece uma concepção mais abrangente da realidade do sistema público de saúde, já que objetiva a excelência no conhecimento da SM, por meio do domínio, gerenciamento e inteiração dos procedimentos internos da RAPS pelos residentes a outras redes e recursos, de modo a fomentar o cuidado integral e o trabalho multidisciplinar. Além disso, esse modelo utiliza uma regionalização municipal que permite aos residentes aprender sobre o desenvolvimento do tratamento nos centros de atenção psicossocial (Caps) para as unidades básicas de saúde (UBS) e acompanhar o atendimento do paciente após alta hospitalar. Assim, o processo de aprendizagem é aprimorado, pois os residentes adicionam diversos conhecimentos à prática, além de melhorar o cuidado com a SM na cidade em questão ${ }^{12}$.

Apesar da atraente estética desse modelo de RM, ele ainda conta com uma série de limitações, como a dificuldade em harmonizar as tarefas acadêmicas com a elevada demanda de transtornos mentais do SUS e a preservação de práticas atualizadas na mesma esfera. Ademais, incorporar os residentes nas rotinas diárias e nas ações de cada unidade de saúde e das consultas unicamente de psiquiatria ainda é um obstáculo desde a formação médica ${ }^{12}$.

Além disso, durante esta pesquisa, constatou-se uma grande discrepância na quantidade de questões sobre SM quando se compararam diferentes instituições. Alguns aspectos podem ser inferidos para tais diferenças, como a importância dada aos transtornos mentais pelos hospitais e pelas instituições de RM e também porque, muitas vezes, as comissões de prova de RM têm representação insuficiente de professores mais afeitos à graduação. Assim, embora o empenho para o desenvolvimento de professores da Faculdade de Medicina nos departamentos e nas instituições de psiquiatria e SM venha aumentando, ainda existem necessidades de desenvolvimento do corpo docente que não foram atendidas, como um tempo exclusivo para tal, oficinas de habilidades e orientação. Contudo, a carreira na educação médica enfrenta algumas barreiras, como a ausência de financiamento e tempo, além das excessivas demandas clínicas ${ }^{14}$.

Para mais, conforme os enfoques observados nas questões de RM, verifica-se uma tendência preponderante em cobrar aspectos biológicos dentro das mais diversas áreas da Medicina. Essa dicotomia é extremamente evidente em áreas como a cirurgia geral, em que nenhuma questão abordando a temática foi encontrada. Essa preponderância, em detrimento de aspectos psíquicos do paciente nos pré e pós-operatórios, por exemplo, contribui para que a Medicina ainda continue sendo predominantemente biomédica, em vez de centrada no sujeito ${ }^{15}$.

Além disso, as questões dentro dos subtemas da SM apresentaram muita discrepância, sendo mais proeminente o tema "psicofarmacologia", com aproximadamente o dobro de questões da segunda temática mais explorada. Diante disso, é possível inferir acerca dos aspectos teóricos valorizados dentro da própria área. Mais uma vez, exploram-se de forma preponderante os aspectos biológicos. A cobrança excessiva de tratamento farmacológico pode fazer com que os clínicos gerais recém-formados, apesar de reconhecerem e diagnosticarem corretamente os transtornos mentais em geral, sintam-se inseguros em prescrever tratamentos não farmacológicos para seus pacientes ${ }^{16,17}$. É importante ressaltar que a SM, especialmente a psiquiatria, representante da área médica, necessita se relacionar com os fatores orgânico e biológico para receber sua credibilidade. Porém, de uma forma a mais, exemplifica-se o olhar centrado no aspecto biológico do sujeito, o que se distancia de aspectos psicossociais aos quais a prática psiquiátrica está associada, e, por conta disso, "objetifica-se o transtorno mental"18,19.

Identificaram-se os transtornos mentais comuns em poucas questões. Os transtornos de ansiedade, por exemplo, foram abordados em apenas $6 \%$ das questões analisadas, apesar de evidências mostrarem um aumento de quase $20 \%$ desses transtornos e de seus impactos de morbidade na população entre 2005 e 2015. Segundo a Organização Mundial da Saúde (OMS), os transtornos depressivos são classificados como o maior contribuinte único para a perda de saúde não fatal, enquanto os transtornos de ansiedade ficam na sexta colocação ${ }^{20}$. No Brasil, os transtornos depressivos e ansiosos correspondem, respectivamente, à quinta e sexta causas de anos de vida vividos com incapacidade ${ }^{21}$.

No que se refere à psiquiatria infantojuvenil, os transtornos somatoformes e os transtornos de regulação estão entre os assuntos mais relevantes na prática rotineira de clínicos e de pediatras ${ }^{19}$. Em nossa pesquisa, verificamos apenas cinco questões acerca desse segundo tema, e apenas duas se referiam à psiquiatria infantojuvenil. Sobre 
transtornos de regulação do sono ou das emoções, não foram encontrados itens em nossa análise. Assim, pode haver uma tendência de os futuros médicos não se aprofundarem nesse tema como deveriam, criando uma lacuna de conhecimento que desfavorece o atendimento integral.

Acerca das habilidades práticas, a relação médicopaciente poderia ser algo muito bem explorado nos cenários de prática da SM. Para pediatras e clínicos, a competência mais relevante na psiquiatria infantojuvenil refere-se à comunicação com crianças e adolescentes e seus familiares, uma vez que esse processo facilita a adesão ao tratamento e um melhor cuidado $^{22}$. Embora essas práticas cruciais sejam avaliadas em alguns concursos de RM por meio da prova prática, esta não é uma realidade para todas as instituições. Daquelas aqui analisadas, sete apresentam essa etapa na seleção.

Como consequência da problemática exposta no enfoque dado à SM nas provas de RM, perpetua-se, na formação médica, uma visão assistencial biologicista, em detrimento do enfoque destinado às questões psicossociais. Com isso, usuários acabam sendo olhados ainda de maneira incompleta pelos médicos generalistas na atenção primária ${ }^{6,13,23}$.

Portanto, se o SUS prega a integralidade como um dos princípios maiores ${ }^{24}$, é fundamental que os médicos generalistas estejam atentos às condições mentais dos pacientes, mesmo em situações não psicológicas, e entendam que sintomas clinicamente inexplicáveis podem ter raízes psicológicas ${ }^{13}$. Cabe a esses médicos a apropriação desse público, entendendo o paciente como um ser multidimensional, facilitando a criação de vínculo, a adesão ao tratamento (farmacológico ou não) e a melhoria na qualidade de vida dos indivíduos, uma vez que há forte relação entre a falta de atenção aos problemas relacionados à SM e o aumento da mortalidade ${ }^{6}$. Além disso, é de extrema importância que se considerem as relações familiares dos pacientes, uma vez que a família contribui para o tratamento dos indivíduos, de maneira positiva ou negativa, dependendo da abordagem que é realizada, já que os familiares consideram o médico generalista o principal profissional responsável por orientar as ações, aconselhar e confirmar as medidas adotadas para o tratamento ${ }^{25}$.

Perante o exposto, de acordo com Rosenthal et al. ${ }^{8}$, Thomas et al. $^{13}$ e Fricchione et al. $^{23}$, quando se considera a etiologia complexa dos transtornos mentais, que pode causar efeitos orgânicos gerais, é necessária uma compreensão eficaz da interação entre fatores biológicos, psicológicos e sociais. Dessa forma, a SM deve ser considerada em todas as disciplinas, não só em psiquiatria, de forma a entender o sujeito, objeto de cuidados médicos, de maneira mais integral. Com isso, pode-se ampliar o desenvolvimento de estratégias de gestão, habilidades de comunicação não estigmatizantes e uma postura não julgadora dos pacientes com algum tipo de sofrimento. Portanto, os generalistas devem ser capacitados para prevenção de SM e intervenção precoce destinada a ela, o que significa focalizar os cuidados primários, abarcar sintomas sutis e subsindrômicos e entregar possíveis resultados do tratamento. Não obstante, a educação médica permite um cuidado melhor da SM dos próprios profissionais em questão ${ }^{13}$.

Portanto, com todos os resultados encontrados neste trabalho, podemos refletir acerca das dificuldades de mudanças concretas no modelo de formação médica, de modo a ampliar o olhar biopsicossocial. A ampliação da carga horária específica para a SM, trazida pelas DCN, representa um esforço inicial. A adaptação dos concursos de RM aos conteúdos fundamentais propostos pelas diretrizes e aos aspectos exigidos pelo SUS, principalmente no que diz respeito à participação em serviços básicos de saúde, é um dos aspectos que dificultam tais modificações ${ }^{26}$.

Como possível extrapolação desses achados, algumas inferências acerca das modificações nos currículos acadêmicos dos cursos de Medicina podem ser vislumbradas, gerando hipóteses para pesquisas futuras. Com a pouca expressão dada à SM nos concursos de RM, é possível que a importância atribuída à psiquiatria e à SM na graduação não seja aquela sugerida pelas DCN. Pelo enfoque biologicista aqui observado, nas questões referentes ao tema, questiona-se se a exposição dos acadêmicos às temáticas e aos cenários de práticas em SM vem sendo congruente àqueles objetivados pelas modificações das $\mathrm{DCN}^{27}$. Tudo isso colabora para a perpetuação de aspectos desfavoráveis à temática. Além disso, agravam-se déficits de conhecimento, exacerbam-se visões distorcidas do cuidado em SM e ampliam-se pensamentos estigmatizadores por partes de estudantes e médicos ${ }^{7,13,28}$. Portanto, ressalta-se a necessidade de novas pesquisas, por meio de nova metodologia, para verificar o seguimento das $\mathrm{DNC}$, em relação à SM, por parte das instituições de ensino superior (IES).

Como limitações desta pesquisa, deve-se alertar que não foi um escopo previsto a análise das provas práticas das instituições selecionadas, sendo as dificuldades de coleta dos dados referentes a essas fases dos concursos os principais motivos para tal. Não obstante, foram encontradas restrições no alcance de provas de RM, na modalidade acesso direto da UEM (de 2009 a 2015), USP-SP (2015), Unicamp (2009) e do SUSSP (2009). Diante disso, buscaram-se essas provas por meio de comunicações eletrônicas diretas com as comissões de RM dessas instituições. A UEM declarou via e-mail que as provas não seriam disponibilizadas, pois são elaboradas pelos docentes com exigências de sigilo, discrição e responsabilidade, e, dessa forma, as questões devem ser utilizadas exclusivamente para os PRM. Já a Unicamp se mostrou aberta à solicitação, mas não 
enviou o arquivo com a prova a tempo do envio deste artigo, enquanto USP-SP e SUS-SP não retornaram.

Apesar disso, ao avaliar o seguimento das DCN pelos concursos de RM, no que tange à SM, este trabalho apresenta dados, expõe questões e traça caminhos. O seguimento das DCN pelos concursos de RM pode ser um caminho para uma avaliação mais equânime entre os candidatos, uma vez que seguirá caminhos nacionais, no lugar de seguir as fortalezas particulares de cada instituição de ensino. Além disso, a aplicação das DCN nos concursos poderá servir tanto como fomentador de mudanças quanto como um indicador. No que tange à SM, uma consequência possível pode ser o aumento de pautas específicas sobre o tema no currículo médico, com ampliação de experiências com pacientes com adoecimento mental e percepção de sofrimento mental em doenças físicas. Espera-se, com isso, a ruptura de estereótipos negativos à população acometida por transtornos mentais, diminuindo impactos da estigmatização ${ }^{7}$.

Diante da dissonância entre a necessidade do sistema de saúde e o conteúdo abordado nos concursos de RM, os cursos preparatórios continuam tendo seu lugar na complementação do ensino de graduação, com objetivo de aprovação na RM. Com conteúdos baseados nos concursos, a valorização de conteúdos de SM e de habilidades práticas ainda não é observada ${ }^{4}$. Se os concursos de ingresso nos PRM não começarem a seguir o preconizado pelas DCN de 2014, dificilmente haverá mudança efetiva nas práticas médicas, no que tange à SM.

\section{CONSIDERAÇÕES FINAIS}

As DCN de 2014, ao transformarem a estrutura do curso de graduação, pretendiam tornar a formação médica mais humanizada, articulada e integrada. $O$ aumento da carga horária em SM, no regime do internato, adveio da relevância desse assunto na prática médica. Apesar da tendência de crescimento nas porcentagens de questões nas provas de RM em algumas instituições, este trabalho observou que os concursos de RM ainda estão muito aquém do preconizado nas DCN. Nenhuma instituição apresentou uma crescente de forma linear após 2014. Além disso, a maior parte das questões apresentadas nos concursos ainda tem enfoque no aspecto biológico em detrimento de aspectos psicossociais. Sendo assim, as provas de RM acabam desconsiderando toda pluralidade de aspectos que a SM engloba, desacreditando em sua importância na prática médica. Com isso, mantém-se um ciclo de pouca consideração de aspectos importantes no currículo médico, com consequências para os estudantes de Medicina, residentes, clínicos e pacientes com transtornos mentais e seus familiares.

\section{CONTRIBUIÇÃO DOS AUTORES}

Felipe Pinheiro de Figueiredo participou da concepção, supervisão e análise formal da pesquisa. Julia Melara e Gustavo Gomedi foram responsáveis pela execução da pesquisa, incluindo curadoria de dados, investigação, metodologia, administração do projeto, assim como a escrita do artigo. Todos os autores revisaram e editaram a versão final do manuscrito. Além disso, Julia Melara ficou responsável pelo processo de aquisição de financiamento e recursos, enquanto Gustavo Gomedi pelo uso de software.

\section{CONFLITO DE INTERESSES}

Declaramos não haver conflito de interesses.

\section{FINANCIAMENTO}

Acadêmica do Curso Medicina, Campus Maringá. Bolsista do Programa Institucional de Bolsas de Iniciação Cientifica PIBIC/ ICETI - Unicesumar.

\section{REFERÊNCIAS}

1. Machado CD, Wuo A, Heinzle M. Educação médica no Brasil: uma análise histórica sobre a formação acadêmica e pedagógica. Rev Bras Educ Med 2018;42(4):66-73. doi: 10.1590/1981-52712015v42n4rb20180065.

2. Gomes AP, Rego S. Transformação da educação médica: é possível formar um novo médico a partir de mudanças no método de ensinoaprendizagem? Rev Bras Educ Med. 2011;35(4):557-66. doi:10.1590/ S0100-55022011000400016.

3. dos Santos RA, Nunes MPT. Medical education in Brazil. Med Teach. 2019;41(10):1106-11. doi: 10.1080/0142159X.2019.1636955.

4. André JC, de Melo JCR, Lima ARA, Brienze SLA, Werneck AL, Fucuta PS. Cursos preparatórios para os exames de residência e a evasão dos cenários de prática: cadê o interno que estava aqui? Rev Bras Educ Med. 2019;43(1):105-14. doi: 10.1590/1981-52712015v43n1 rb20170127ing.

5. Brasil. Resolução no 3, de 20 de junho de 2014. Institui Diretrizes Curriculares Nacionais do Curso de Graduação em Medicina e dá outras providências. Diário Oficial da União; 20 jun 2014. p. 8-11 [acesso em 11 mar 2020]. Disponível em: http://portal.mec.gov.br/index. php?option=com_docman\&view=download\&alias=15874-rces003 14\&category_slug=junho-2014-pdf\&ltemid=30192.

6. McCarron RM, Bourgeois JA, Chwastiak LA, Folsom D, Hales RE, Han J, et al Integrated medicine and psychiatry curriculum for psychiatry residency training: a model designed to meet growing mental health workforce needs. Acad Psychiatry. 2015;39(4):461-5. doi: 10.1007/s40596-015-0348-3.

7. Oliveira AM, Machado D, Fonseca JB, Palha F, Moreira PS, Sousa N, et al. Stigmatizing attitudes toward patients with psychiatric disorders among medical students and professionals. Front Psychiatry. 2020;11:326-32. doi: $10.3389 /$ fpsyt.2020.00326.

8. Rosenthal RH, Levine RE, Carlson DL, Clegg KA, Crosby RD. The "shrinking" clerkship: characteristics and length of clerkships in psychiatry undergraduate education. Acad Psychiatry. 2005; 29(1):47-51. doi: 10.1176/appi.ap.29.1.47.

9. Warnke I, Gamma A, Buadze M, Schleifer R, Canela C, Strebel B. predicting medical students' current attitudes toward psychiatry, interest in psychiatry, and estimated likelihood of working in psychiatry: a crosssectional study in four European countries. Front Psychiatry. 2018 Mar 12;9:49-61. doi: 10.3389/fpsyt.2018.00049. 
10. Brasil. Decreto $n^{\circ} 80.281$, de 5 de setembro de 1977. Brasília: Câmara dos Deputados; 1977 [acesso em 10 jul 2020]. Disponível em: https://www2. camara.leg.br/legin/fed/decret/1970-1979/decreto-80281-5-setembro1977-429283-normaatualizada-pe.pdf.

11. Brasil. Resolução $n^{\circ} 2$, de 27 de agosto de 2015. Adequa a legislação da Comissão Nacional de Residência Médica ao art. 22 da Lei 12.871/2013, acerca do processo de seleção pública dos candidatos aos Programas de Residência Médica. Diário Oficial da União; 28 ago 2015. Seção 1, p. 31 [acesso em 10 jul 2020]. Disponível em: https://www.in.gov.br/materia/-/ asset_publisher/Kujrw0TZC2Mb/content/id/32425105/do1-2015-08-28resolucao-n-2-de-27-de-agosto-de-2015-32425068.

12. Generoso MB, Mazaferro PN. Integrated residency in psychiatry: an innovative model of medical training in Brazil. Acad Psychiatry. 2019;43(5):551-2. doi: 10.1007/s40596-019-01102-1.

13. Thomas S, Pai N, Dawes K, Wilson C, Williams V. Updating medical school psychiatry curricula to meet projected mental health needs. Australas Psychiatry. 2013;21(6):578-82. doi: 10.1177/1039856213500092.

14. De Golia SG, Cagande CC, Ahn MS, Cullins LM, Walaszek A, Cowley DS. Faculty development for teaching faculty in psychiatry: where we are and what we need. Acad Psychiatry. 2019;43(2):184-90. doi:10.1007/s40596018-0916-4.

15. Ribeiro MMF, Amaral CFS. Medicina centrada no paciente e ensino médico: a importância do cuidado com a pessoa e o poder médico. Rev Bras Educ Med. 2008;32(1):90-7. doi: 10.1590/S0100-55022008000100012.

16. Picco L, Seow E, Chua BY, Mahendran R, Verma S, Chong SA, et al. Recognition of mental disorders: findings from a cross-sectional study among medical students in Singapore. BMJ Open. 2017 Dec 21;7(12):e019038. doi: 10.1136/bmjopen-2017-019038.

17. Zafra-Tanaka JH, Pacheco-Barrios K, Inga-Berrospi F, Taype-Rondan A. Selfperceived competencies in the diagnosis and treatment of mental health disorders among general practitioners in Lima, Peru. BMC Med Educ. 2019;19(1)464-71. doi: 10.1186/s12909-019-1900-8.

18. D'Angelis RM. Psiquiatria em análise: narrativas da prática psiquiátrica no SUS [dissertação]. Campinas: Universidade Estadual de Campinas; 2017 [acesso em 2 ago 2020]. Disponível em: http://repositorio.unicamp.br/ jspui/handle/REPOSIP/330297.

19. Lempp T, Heinzel-Gutenbrunner M, Bachmann C. Child and adolescent psychiatry: which knowledge and skills do primary care physicians need to have? A survey in general practitioners and paediatricians. Eur Child Adolesc Psychiatry. 2016;25(4):443-51. doi: 10.1007/s00787-015-0757-6.
20. World Health Organization. Depression and other common mental disorders: global health estimates. Geneva: WHO; 2017 [acesso em 28 jul 2020]. Disponível em: https://www.who.int/mental_health/management/ depression/prevalence_global_health_estimates/en/.

21. Lopes CS. Como está a saúde mental dos brasileiros? A importância das coortes de nascimento para melhor compreensão do problema. Cad. Saude Publica. 2020;36(2):e00005020. doi: 10.1590/0102-311×00005020.

22. Jung HY, Kim JW, Lee $\mathrm{S}$, Yoo SH, Jeon JH, Kim TW, et al. A study of core humanistic competency for developing humanism education for medical students. J Korean Med Sci. 2016;31(6):829-35. doi: 10.3346/ jkms.2016.31.6.829.

23. Fricchione GL, Borba CPC, Alem A, Shibre T, Carney JR, Henderson DC. Capacity building in global mental health: professional training. Harv Rev Psychiatry. 2012;20(1):47-57. doi:10.3109/10673229.2012.655211.

24. Brasil. SUS: princípios e conquistas. Brasília: Ministério da Saúde, Secretaria Executiva; 2001 [acesso em 15 jul 2020]. Disponível em: https://bvsms. saude.gov.br/bvs/publicacoes/sus_principios.pdf.

25. Riebschleger J, Scheid J, Luz C, Mickus M, Liszewski C, Eaton M. How are the experiences and needs of families of individuals with mental illness reflected in medical education guidelines? Acad Psychiatry. 2008;32(2):119-26. doi: 10.1176/appi.ap.32.2.119.

26. de Almeida MJ, de Campos JJB, Turini B, Nicoletto SCS, Pereira LA, Rezende LR, et al. Implantação das Diretrizes Curriculares Nacionais na graduação em Medicina no Paraná. Rev Bras Educ Med. 2007;31(2):156-65. doi: 10.1590/S0100-55022007000200006.

27. Brasil. Portaria $n^{\circ} 3.088$, de 23 de dezembro de 2011. Institui a Rede de Atenção Psicossocial para pessoas com sofrimento ou transtorno mental e com necessidades decorrentes do uso de crack, álcool e outras drogas, no âmbito do Sistema Único de Saúde (SUS). Brasília: Ministério da Saúde; 2011 [acesso em 27 jul 2020]. Disponível em: http://bvsms.saude.gov.br/ bvs/saudelegis/gm/2011/prt3088_23_12_2011_rep.html.

28. Malhi GS, Parker GB, Parker K, Carr VJ, Kirkby KC, Yellowlees P, et al. Attitudes toward psychiatry among students entering medical school. Acta Psychiatr Scand. 2003;127(1):424-9. doi: 10.1034/j.1600-0447.2003.00050.x. 